\title{
Teaching Visual Storytelling for virtual production pipelines incorporating Motion Capture and Visual Effects
}

\author{
Gregory Bennett* \\ Auckland University of Technology
}

\author{
Jan Kruse K $^{\dagger}$ \\ Auckland University of Technology
}

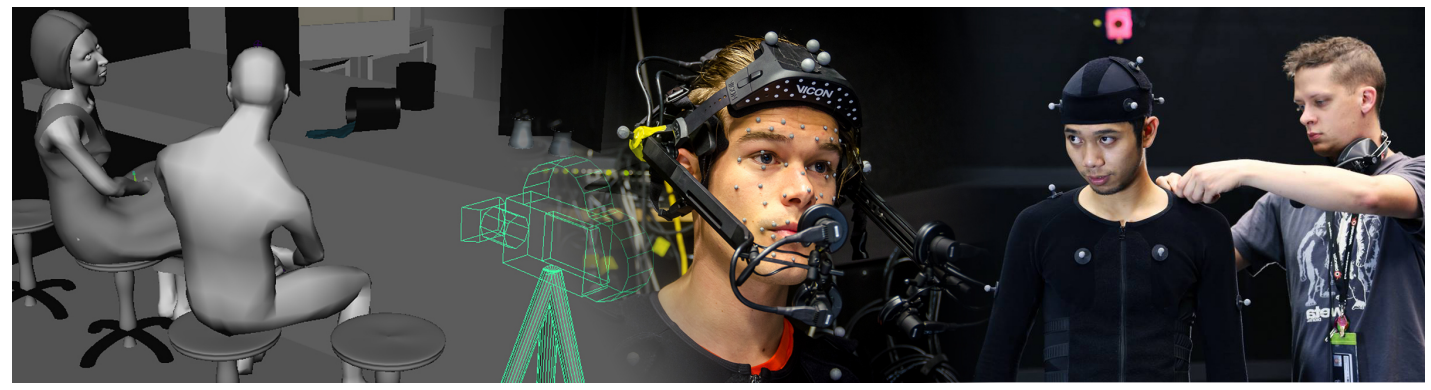

Figure 1: Performance Capture for Visual Storytelling at AUT.

\begin{abstract}
Film, television and media production are subject to consistent change due to ever-evolving technological and economic environments. Accordingly, tertiary teaching of subject areas such as cinema, animation and visual effects require frequent adjustments regarding curriculum structure and pedagogy. This paper discusses a multifaceted, cross-disciplinary approach to teaching Visual Narratives as part of a Digital Design program. Specifically, pedagogical challenges in teaching Visual Storytelling through Motion Capture and Visual Effects are addressed, and a new pedagogical framework using three different modes of moving image storytelling is applied and cited as case studies. Further, ongoing changes in film production environments and their impact on curricula for tertiary education providers are detailed, and appropriate suggestions based on tangible teaching experience are made. This paper also discusses the advantages of teaching Motion Capture in the context of immersive environments.
\end{abstract}

Keywords: motion capture, visual storytelling, narrative, visual effects, curriculum

\section{Outline and Background}

Visual Storytelling has been used in various contexts, from marketing [Mileski et al. 2015] to film [McClean 2007] and even games [Howland et al. 2007]. While the Digital Design program at Auckland University of Technology utilizes Visual Narratives in different contexts throughout the curriculum, the focus of this paper is on film and immersive environments.

Teaching Visual Storytelling or Visual Narratives poses significant challenges to educators and students alike. The topic requires a

\footnotetext{
*e-mail:gbennett@aut.ac.nz

†e-mail:jkruse@aut.ac.nz
}

Permission to make digital or hard copies of part or all of this work for personal or classroom use is granted without fee provided that copies are not made or distributed for commercial advantage and that copies bear this notice and the full citation on the first page. Copyrights for third-party components of this work must be honored. For all other uses, contact the Owner/Author.

Copyright is held by the owner/author(s).

SA'15 Symposium on Education, November 02 - 06, 2015, Kobe, Japan.

ACM 978-1-4503-3927-8/15/11.

http://dx.doi.org/10.1145/2818498.2818516 solid theoretical foundation, and could traditionally only be explored through theory and examples in a lecture/lab style context. Particularly programs that aim to deliver content in a studio-based environment suffer from the complexity and cost-time-constraints inherently part of practical inquiry into storytelling through short film production or visual effects animation. Further, due to the structure and length of Film, Visual Effects and Digital Design degrees, there is normally only time for a single facet of visual narrative to be addressed, for example a practical camera shoot, or alternatively a visual effects or animation project. This means that comparative exploratory learning is usually out of the question, and students might only take a singular view on technical and creative story development throughout their undergraduate years. Normally only postgraduate study offers sufficient time to understand and explore multiple facets of filmic narratives.

Film production pipelines in general, and visual effects workflows in particular, have seen a significant paradigm-shift in the past decade. Traditional workflows are based on frequent conversions between two dimensional images and three dimensional data sets [Okun and Zwerman 2010]. For example, the acquisition of camera images (digital or on film) projects a 3D world through a lens on to a 2D camera plane (sensor or film back). This digitized 2D image is then used to match-move the camera, effectively reconstructing a $3 \mathrm{D}$ scene from $2 \mathrm{D}$ data. The $3 \mathrm{D}$ scene is manipulated as needed, and then rendered back into a sequence of $2 \mathrm{D}$ images, which finally make their way into compositing software. One can see the redundancy within these conversions, but technological limitations and established software tools, data formats and workflows simply dictate the way film (effects) production is traditionally accomplished. Recent developments in the fields of RGB-D cameras [Hach and Steurer 2013], performance capture, deep compositing, real-time stereoscopic rendering as well as scene based file formats such as FBX (filmbox) or ABC (alembic) start to revolutionize the way pipelines in visual effects studios are set up. Less conversions and a strong emphasis on 3D data sets instead of images have changed workflows and simplified interchange of image data. Within their undergraduate years, students are usually not exposed to the changes in production environments. Students experience a snapshot of current technology, and therefore lack an appreciation and understanding for the implications and benefits of technological change.

One of the most significant workflow changes in commercial environments is probably the way visual storytelling has been detached 
from a linear production mode. Using techniques such as performance capture allows the decoupling of performance from cinematography. While the acting is recorded in form of a motion and facial capture, the 'virtual camera' can be changed at a later stage during production. This is a seemingly obvious, but important difference to traditional image-based filming. It allows film makers to employ a faster and less restricted construction of the narrative through acting or performance, with the possibility to alter environments, camera, lights and visual style at any point of the subsequent production pipeline. This impacts not just on abstract or hyperrealistic visual styles, but also on invisible and seamless visual narratives as classified by McClean [2007]. But more importantly, it has some significant implications for teaching the fundamentals of cinematic storytelling. Cinematic or visual storytelling is the basis of any practical moving image teaching whether for live action, animation or visual effects production. Understanding and practically applying the conventions of cinematography (shot size, framing, camera movement, lighting), editing (shot coverage, continuity and montage editing styles, point-of-view, screen direction, cutting on action) and sound (diegetic/non-diegetic audio) remains a core pedagogical challenge and goal in learning outcomes for students. The taxonomy of these conventions remain the core of foundational film studies [Bordwell and Thompson 2008; Corrigan and White 2012; Kolker 2005] and film making texts [Bowen 2013; Katz 1991; Sijll 2005], and provide both useful frameworks and practical challenges for their application within curriculum design and delivery.

Recent developments in digital production technologies and tools have transformed the possibilities for both technical pipelines and the creative development of visual storytelling. The term 'virtual production' has been coined to describe a confluence of technologies including motion capture (MoCap), gaming software and virtual cinematography. Improved live 3D data streaming, which has enabled an increasingly seamless, realtime $3 \mathrm{D}$ production pipeline from conception to delivery of animation and visual effects moving image, has been hailed as 'The New Art of Virtual Moviemaking'[Autodesk 2009].

The increasing establishment and utilisation of MoCap studios where the 3D tracking of performers has been augmented by the ability to view MoCap data re-targeted live to digital characters in virtual sets via a hand-held live-tracked virtual camera, has allowed for a more direct, 'hands-on' and user-friendly engagement and interaction of directors, cinematographers and actors in realtime within the physical space of a live studio environment, without recourse to the mediation of 3D animators. Performance action can also be captured initially, with cinematographic choices applied flexibly at any point subsequently, allowing for the potentially limitless creative exploration of framing, camera movement and editing options. This convergence of the immediacy of live action shooting conditions with more creative and interactive accessibility to the digital post-production process has seen the $3 \mathrm{D}$ previsualization (previz) environment become a key testing ground and developmental tool for exploring and maximising effective visual storytelling.

There has also been an expansion in the possibilities for the language of visual storytelling with the continuing utilisation, development and technical innovation in digital visual effects cinema, and increased discourse around the status of visual effects content within cinematic narrative, whether dismissed as mere digital 'spectacle', or acknowledged as an integral and often innovative feature of the ongoing development of cinematic language. In particular, the potentially infinite flexibility of the virtual camera, unchained from the real-world constraints, and the influence of newer media forms such as gaming, virtual reality and interactive graphic interfaces continues to expand and inform the grammar of visual storytelling within the film form.

\section{Identifying pedagogical needs in rapidly changing environments}

Here in the Digital Design Department, in the School of Art \& Design, Auckland University of Technology (AUT) in New Zealand, our undergraduate programme caters to a largely vocationally oriented cohort who can select pathways in Visual Effects, 3D Animation and Game Design. These areas encourage overlaps and convergences according to the interests and aspirations of individual students. A recently commissioned 24-camera Motion Analysis MoCap studio at AUT (including a Vicon Cara Facial MoCap system and an Insight VCS virtual camera) has allowed for the introduction of a comprehensive MoCap minor programme, covering all aspects of the MoCap pipeline including capture, data processing and post-production. In relation to our established pathways MoCap has been found to be a valuable augmentation to all of the disciplines, and often a point of convergence in issues of performance (digital characters for animation, gaming or visual effects driven by MoCap or key-frame animation or a combination of both) and visual storytelling (3D previz for animation, gaming or visual effects).

This paper focuses on the construction of a foundational lesson for teaching visual storytelling for a visual effects sequence using MoCap. It identifies some of the key factors of teaching and learning strategies based on practical experience with delivering the fundamentals of a film grammar curriculum. The lesson plan incorporates new teaching strategies in acknowledgement of the changing digital production environment, and the evolving language of visual effects cinema. This lesson plan is based on the creation and exploration of the creative possibilities for visual storytelling in a 3D previz sequence, and is designed for students new to the Motion Capture pipeline , but who have some foundational knowledge of the primary 3D software platform used, in this case Autodesk Maya.

Teaching strategies are based on both our experience with teaching the rules of filmic language (grounded in shooting and editing live action), and our experience with 3D animation and visual effects as expanding and informing the lexicon of live action cinema expression. In addition advice from industry advisors of skillset requirements are factored in, in particular the need for graduates to understand the visual narrative context of a particular element, whether a VFX shot, animation or MoCap performance. From this a set of learning outcomes are formulated based on the following guiding factors:

- Focus on core visual storytelling principles despite changing digital tools

- Students need to understand the 'rules'of conventional continuity shooting and editing before breaking them [Bowen 2013]

- Acknowledge changes/developments/possibilities in the grammar of digital moving image storytelling

- Embrace the challenges of creating a successful visual effects sequence i.e. How to make it believable and emotionally involving for audiences, but also harness its 'spectacular'possibilities without recourse to mere 'eye candy'

- Consider advice from industry advisors, namely that they are looking for graduates who understand the cinematographic storytelling context of an animation or visual effects shot, or a MoCap motion editing task 


\section{Lesson Plan}

The following sections outline an approach that has been successfully implemented as part of the Digital Design curriculum. A detailed view of each component of our pedagogical framework, in particular the assignment, brief on Motion Capture, and three editing / exploration approaches as part of the students'studio-based inquiry are given. This brief is a foundational Motion Capture project for students, and is positioned in the second half of their first year of study in three-year undergraduate program. It constitutes the first paper of four, which collectively form a 'Minor' program, which runs throughout the three-year undergraduate course. This sits alongside the Digital Design 'Major', which consists of core studio practice pathways (animation, visual affects and game design), and theory and context studies (See figure 2).

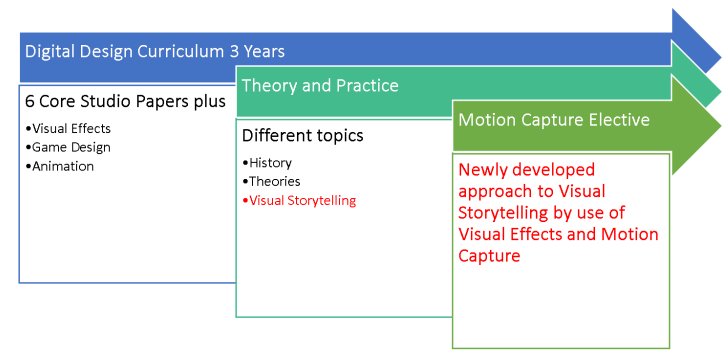

Figure 2: Overview of existing curriculum with added new teaching approach (in red)

\subsection{Assignment Brief}

The specific assignment brief involves producing three versions of a 3D previz sequence based on a 1-page script sample involving two characters. In this example a scene from the screenplay of Godzilla (1998) was adapted. The scene begins with a conversation between and male and female character in a diner. Sounds of the approaching monster interrupt the conversation followed by commotion and mounting destruction viewed through the diner windows. The male protagonist then ventures out to the street to be confronted and subsequently trampled by the monster. This simple scenario provided a conventional set-up, which then builds to a climax with the possibility to explore a more spectacular visual storytelling mode. All of the 3D environment and character assets were pre-supplied for the students so the technical focus is on initially capturing MoCap action, and then focussing on virtual cinematography and editing. Texturing and lighting are disregarded to optimise rendering times for prompt visual feedback.

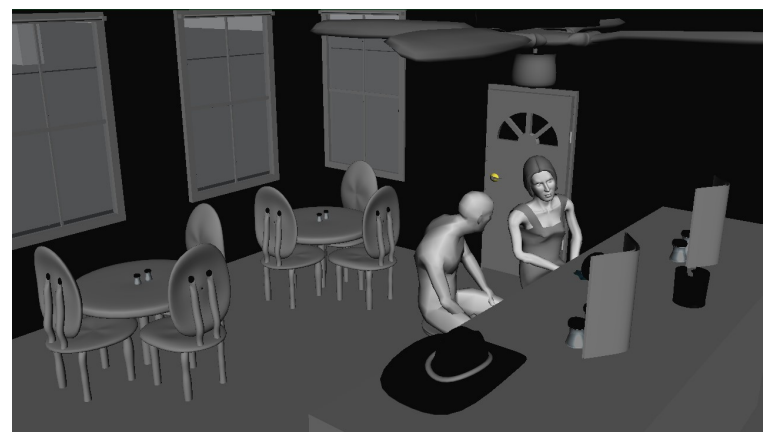

Figure 3: 3D environment: interior of diner with digital characters

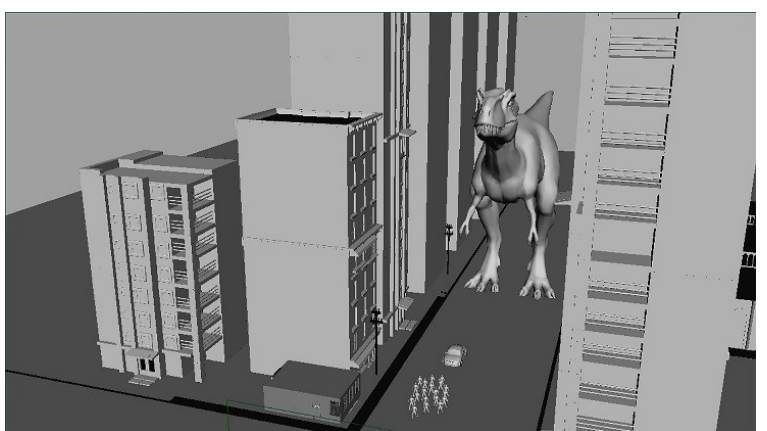

Figure 4: 3D environment: street view with approaching monster

\subsection{Motion Capture}

All of the students work from the same script and set of 3D assets and work in small production groups of 4-5 for the MoCap sessions to capture the required character action.

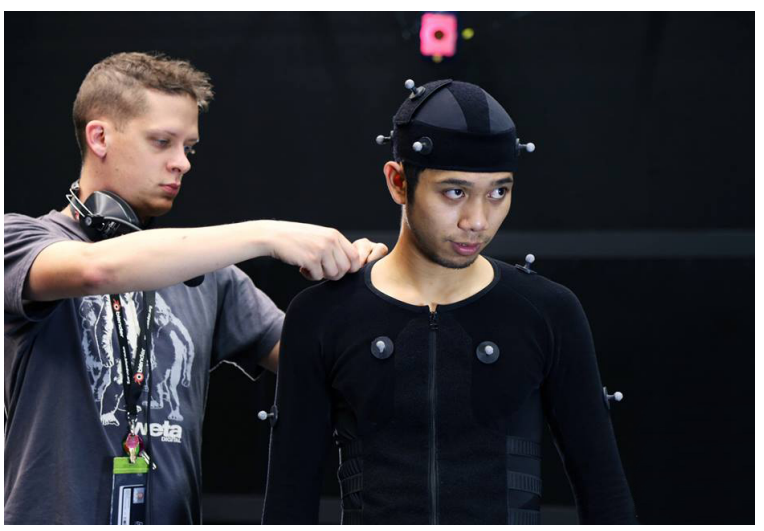

Figure 5: Applying MoCap markers to performer's suit

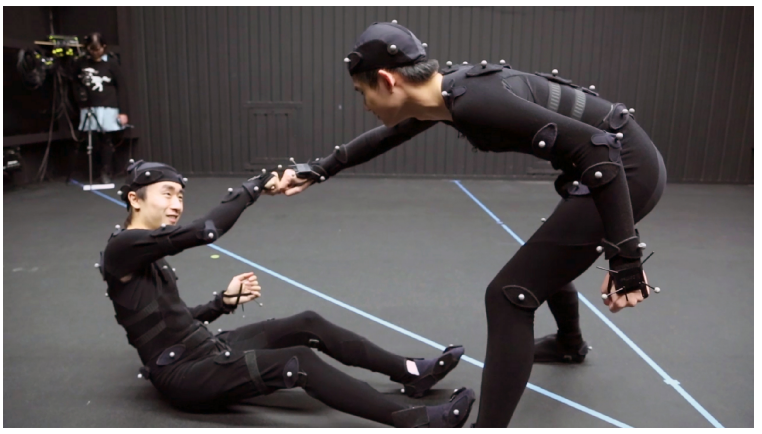

Figure 6: Capturing the performance

\subsection{Difference to traditional cinematography}

For capturing MoCap data the concept of 'shooting to edit', a mantra for teaching traditional live action film shooting protocols, no longer applies. Rather than making on-set framing and camera movement decisions, or actors playing to a particular camera, motion capture uses an 'omniscient frame' [Delbridge 2015] whereby recording of performance is not delineated by a "window typified by the cinematic frame' [Delbridge 2015], but becomes a volumetric operation 'enabled by the capacity of a Motion Capture system's 
camera array to see within a volume, to capture not just the height and width of the 2D frame, but to capture depth (via movement) as well'[Delbridge 2015]. Cinematography becomes a significantly more malleable creative tool within the 3D environment with which to flexibly and non-destructively explore visual storytelling permutations post-performance.

Planning for appropriate shot coverage (typically the master-shot technique: shooting the entire scene in long shot, then re-shooting mid-shots and close-ups and cutaways with actors required to repeat performances each time) is no longer required, and performance capture times can be significantly faster than shooting the same action as conventional single camera live action shooting. Lighting and on-set continuity is also no longer a necessity.

MoCap is a relatively quick way of obtaining moving figures/performers as 3D assets. The relatively recent introduction of the Human IK feature in Autodesk Maya and Motion builder has also enabled a more seamless pipeline flow from Motion Builder (where Mocap data is retargeted to a digital character rig) to Maya, and has automated the previously lengthy task of creating bipedal skeletons and creating animation control rigs. In addition the Autodesk Character Generator, which provides fully-rigged, MoCapready and downloadable generic but customisable digital characters, has eliminated the time-intensive labour to make suitable digital characters for previz.

After capture the students work individually on MoCap data cleanup and re-targeting to generic digital characters in Autodesk Maya. The characters are then inserted into the prepared 3D environment and any additional required key-frame animation is added. They then must produce three different edits of the same action utilizing the virtual camera, engaging in three distinct visual language modes. By framing and editing individually the class ends up with many variations from the same source material. This is an extremely useful comparative tool for analysing and discussing how camera and editing can shape action in many different ways, and can also highlight the variables in skill application and industriousness of the students themselves.

This exercise also adds a studio-based teaching approach to what has previously been delivered formally in theory and context classes, where the principles and conventions of cinematography and editing are learnt through written shot-by-shot close-reading analysis of existing film clips. The virtual production environment affords a practical exploration of this territory, improving students'conceptual and contextual skills by virtue of engaging with a flexible 3D environment where exploration and iterative testing of cinematic storytelling principles can be undertaken with relative ease.

A key paradigm in the building blocks of film language is the point-of view (POV) whereby the 'audience is placed into the shot from the character's point-of-view [and] is encouraged to do the thinking and feeling for the character or as the character more directly'[Bowen 2013]. When conceiving and planning a visual effects sequence for the first time we have observed that students will often instinctively frame action in long shot without regard or awareness of the function of POV in facilitating audience engagement. Assignment briefs which direct students to actively consider POV have resulted in sequences in which action is structured more coherently. For example inclusion of simple shot patterns such as close-up + POV + reaction shot lend meaning and significance to visual effects action which otherwise could register as mere 'empty spectacle'.

\subsection{Edit 1}

Creative goal: Create an edited sequence in which the portrayal of the action is structured around the point-of-view of the central protagonist with the aim of creating maximum audience identification and empathy with this character while maintaining the rules and conventions of the continuity style.

Technical constraints: Engage with conventions of continuitystyle shooting and editing in framing, shot sizes, camera movement, point-of-view and reaction shots, shot-reverse-shots, cutaways, continuity of screen direction, cutting on action and other aspects.

Virtual camera behaviour must adhere to and mimic the physical qualities and limitations of a live action shoot. Camera must be:

- mounted on a tripod with either static shots, or pan or tilts, camera height limited by regular tripod height

- mounted on a dolly, tracking shots (can be combined with tripod and tilt)

- mounted on a gib arm/crane limited by the mechanical constraints of a gib arm or camera crane apparatus

To assist students in this the Maya camera is constrained to simple, workable and keyframeable modeled and rigged camera mounts which can be utilised in the 3D scene.

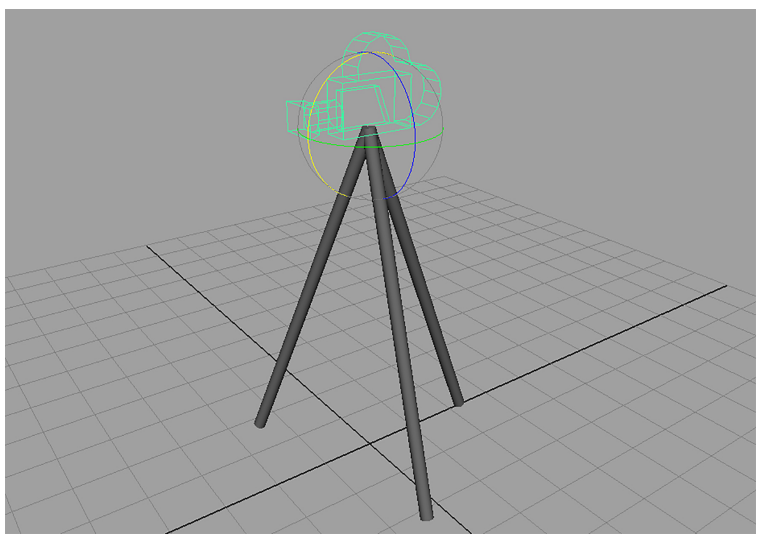

Figure 7: Tripod set-up for Maya camera

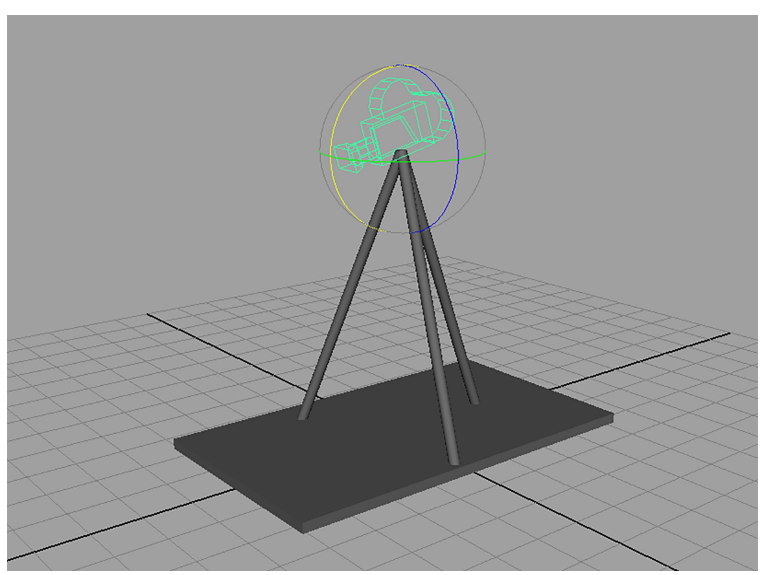

Figure 8: Simple Dolly and Tripod set-up for Maya camera

The virtual camera focal length setting must also reflect real-world camera settings. Students are instructed to adjust the default focal length setting in the Maya camera attribute settings to gain an awareness of how this setting can shape the subjective content of a shot. Focal Length settings are limited to Standard $50 \mathrm{~mm}$, Wide angle $28 \mathrm{~mm}$, Telephoto $90 \mathrm{~mm}$ for this edit. The focal length setting is also activated in the Heads Up Display so that it is visible 


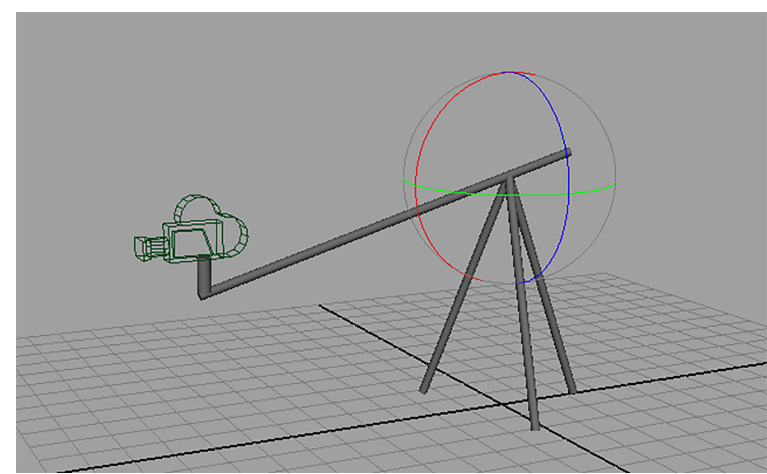

Figure 9: Gib-Arm set-up for Maya camera

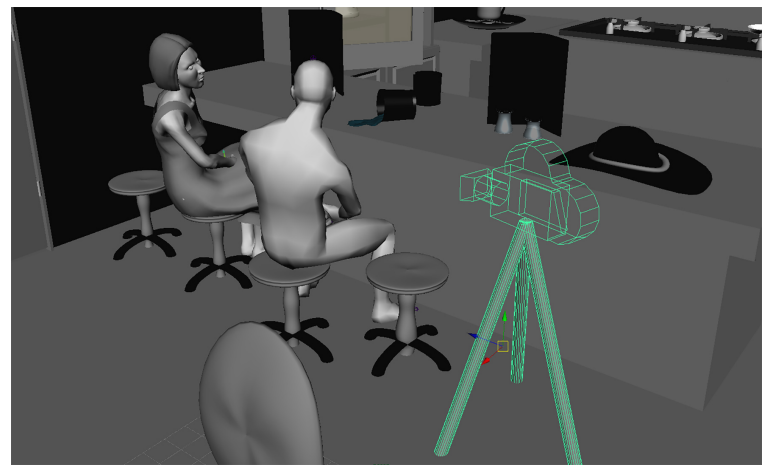

Figure 10: Camera placement in Maya scene

in the frame as reference.

These creative constraints are a useful 'straightjacketing'framework in which students have to more consciously consider shot choices and the staging of the action. The following are some examples of the application of these principles.

Typically the initial two-person conversation is covered with a shot-reverse-shot pattern, followed by point-of-view and reaction shots of the action of the approaching monster occurring outside the diner windows, drawing the audience to the protagonist's view point.

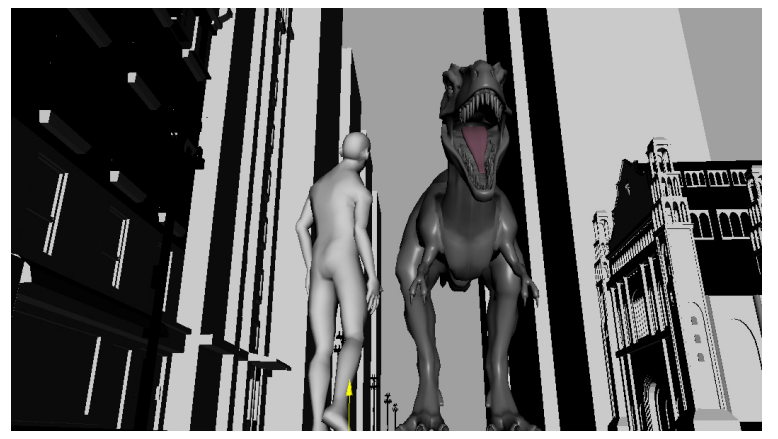

Figure 11: Wide angle 28mm focal length setting

Focal length settings for the same camera position are also able to be explored for their expressive potential.

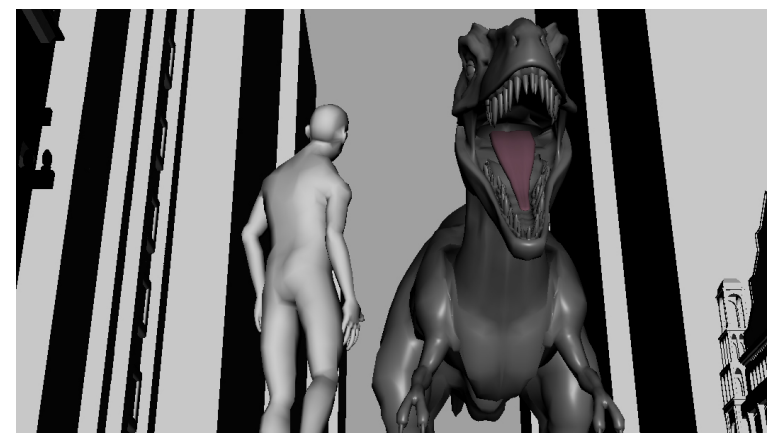

Figure 12: Standard 50mm focal length setting

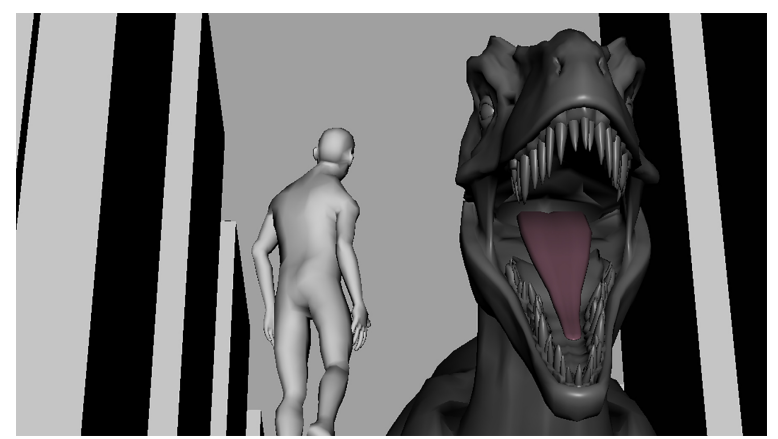

Figure 13: Telephoto 90mm focal length setting

\subsection{Edit 2}

The virtual camera can extend beyond limitations of a live action real-world constraints to include 'impossible'shots. These shots may extend outside of POV reaction shot patterns to include long takes, extreme high or low angles, 360 degree camera tracking, and 'impossible'camera moves.

Experimentation and informed 'breaking of the rules'are encouraged here, while preserving an awareness of the need for audience identification and empathy with character. Camera movement must also remain controlled and purposeful, so control of pace and trajectory through key-framing and animation curves using the graph editor is essential.

Conceptually the camera can also take on a more active POV, and adopt more agency as a conduit for the director's eye, and for the framing and staging of spectacular action. This approach can take on a more self-reflexive mode where the construction of the visual narrative itself can more consciously draw attention to itself. This contrasts the idea of serving continuity with the goal to efface technique in the service of a seamless and 'invisible'cause-and-effect flow of action.

In this context it is possible to explore what Aylish Wood characterizes as the 'expanded narrative space'[Wood 2007] of digital effects cinema. She describes conventional cinema as often centered around characters whom both 'remain a key point of reference for viewer interaction'and 'provide orientation for the space of the image'[Wood 2007]. Visual effects however can offer possibilities for filmic space to become a more active element in itself, even dislocated from its comprehension through character within the continuity system. It can become an 'element which competes with the human figures for the attention of the viewer'[Wood 2007], a dynamic space in which visual effects elements such as the tornados in Twister (1996), the ship in Titanic (1997), and bullet time in The Matrix (1999) become 'vehicles of narrative and not simply 


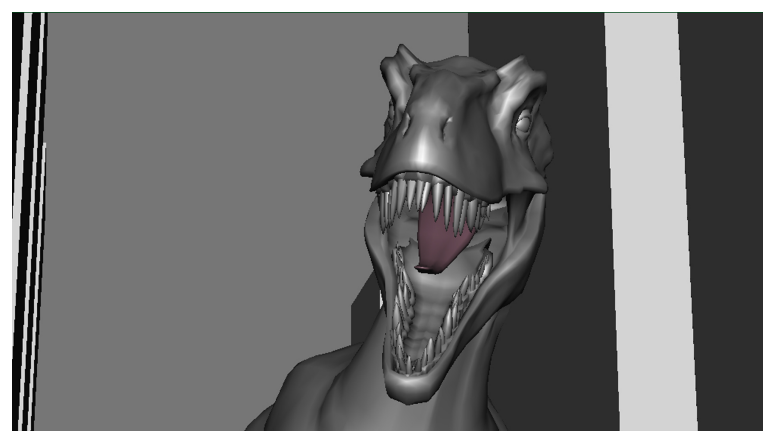

Figure 14: Visual effect as character: Monster Close-up/reaction shot

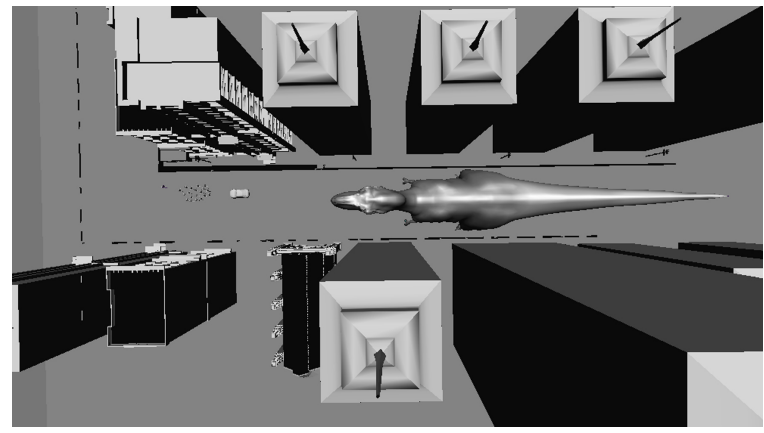

Figure 15: Extreme high angle shot

supportive spaces' [Wood 2007] for character.

\subsection{Edit 3}

The final area of inquiry for the students is Virtual Reality (VR). While VR is oftentimes still considered a very different field, similar to Gaming or Augmented Reality, there have been recent developments to create films in VR environments, with the strong support of Oculus and its latest release Oculus Story Studio [Rusli 2015]. Breaking the link between linear storytelling, cinematography, performance and ultimately the act of consumption by an audience, is a further extension of the 'Edit 2'scenario, and literally breaks with any tradition embedded in visual storytelling. Yet, principles employed by linear narratives might still be required, and the foundational understanding of these principles leads to an advanced artistic practice, which we consider an important learning outcome.

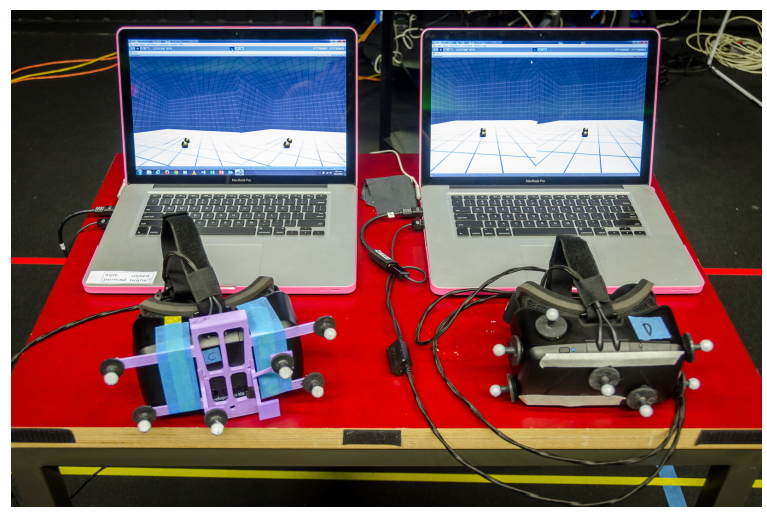

Figure 16: Two Oculus Rift VR headsets ready with markers
Conceptually, the camera takes virtually any perspective that the viewer demands during the consumption of the pre-recorded performance. Utilizing one or multiple Oculus Rift VR head-mounted displays, the students explore this emerging form of narrative, where an engaging performance and focus on environment, light and environmental details gain even higher importance.

While the idea of using VR headsets in conjunction with motion tracking devices is not entirely new [Plemmons and Holz 2014], combining the freedom of Motion Capture with Unity3D for filmic exploration adds an additional level of complexity for the students. Utilizing a custom set-up in the Motion Capture lab whereby the Oculus Rift VR headset worn by a user is tracked live via motion capture markers and streamed into a scene in the Unity game engine, which in turn is streamed live back to the headset viewer as a 3D stereoscopic environment, students are able to experience their previs sequence as a live 'walk-around'virtual environment. This staging presents a whole new set of possibilities and challenges for constructing and experiencing visual narrative in territory which represents a new hybrid frontier in moving image storytelling, and which draws on elements of live cinema, animation, visual effects, gaming, and even theatrical performance paradigms.

Here the first-person POV is entirely privileged, and a level of immersion and immediacy, which impacts the spectator's entire field of vision and bodily orientation, fundamentally intensifies the possible experiential effect for an audience. Without recourse to the 'cut' as it operates in a conventional linear edited sequence, we have observed a number of questions initially arising around the narrative construction possibilities for VR in the testing of the previs scene in a VR platform:

- Viewer agency and spatial control moving from one location to another without inducing a disorientating physical effect on the viewer how do we replace or account for the cut and dissolve as conventions of linear cinematic language?

- Viewer agency and temporal control a sequence can be experienced as playing out in real-time from the first-person POV of the spectator, but exploring a scene through repeated playback from different angles/positions can be a way of embedding additional and potentially richer narrative content.

- Viewer agency and the harnessing of gaming elements such as interactivity in transferring a previs sequence from Maya to Unity with its native set of interactive tools the question of adding interactive elements common to gaming naturally arises, and the possibility for a more complex and seamless hybrid narrative form to emerge incorporating cinematic and gaming elements.

This final exploration of alternative emerging visual narratives, enables the students to understand additional technological and conceptual issues, based on the foundations laid in both previous exercises.

\section{Discussion}

In this section of the paper, we discuss the evaluation of our approach, and point out a few additional details about the third, the immersive approach to teaching visual storytelling.

\subsection{Evaluation of student success}

In order to qualify whether this new approach of adding Motion Capture and visual effects as a means to teach visual storytelling, and evaluate its success with students, we have collected student feedback and also started gathering enrolment data. Currently, the students can choose Motion Capture as an elective topic, as men- 
tioned above. So far, student numbers have nearly doubled within a year, and the course had to be split in order to facilitate the large number of additional participants. Based on our experience, increase in student numbers is largely based on word of mouth as well as relevance of the topic. We believe that a significant increase in student numbers reflects a successful programme. We will continue to monitor the numbers and use this as a measure in future evaluations.

Further, student feedback has been overwhelmingly positive. We asked students whether the combined Motion Capture and visual effects approach would provide any measurable benefit over theory classes and the majority indicated that the exploratory idea helped them to not only develop an understanding of different visual storytelling ideas, but also enabled them to advance their core studio project (as part of the compulsory papers) and develop new ideas in a short timeframe. Research has shown that lectures are oftentimes less effective than group work or studio-based exploration, often coined 'Active Learning' [Meyers and Jones 1993], and it seems that we can confirm this so far, but will continue to evaluate the success of this framework.

\subsection{Immersive Environments}

With the advent of technologies such as easily configurable and usable game engines (e.g. Unreal Engine and Unity3D) and affordable Virtual Reality devices (e.g. Google Cardboard, Oculus Rift), teaching storytelling for immersive environments as part of a Digital Design program, has become viable and offers an interesting additional layer of complexity. While the Creative Game Design pathway at Auckland University of Technology allows student to engage with non-linear storytelling in an interactive context, we decided to add visual storytelling in the wider context of immersive environments to our combined Motion Capture and visual effects pathway. Students capture the performance of one or many actors without committing on a particular camera move, and focus entirely on directing the actors. Then this performance data set is taken into Unity3D where multiple Oculus Rift are used as output devices. This opens up two different viewpoints.

First, the student has to explore and evaluate through the eyes of an audience that is not bound to any particular angle or field of view. Accordingly, considerations regarding the (virtual) environment have to be made.

Second, the student learns to shift focus from directing the whole scene to emphasizing on performance, but more importantly on a captivating story. The main reason is the freedom audiences have in immersive environments. The viewer does not have to look through the lens of the camera, but is free move and look were they want. This requires the student to provide a narrative that ensures the viewer is tempted to follow the story, instead of just looking anywhere.

Therefore, while offering more exploratory freedom, immersive environments help to focus students on the importance of engaging narratives as well.

\section{Conclusion}

Motion Capture in conjunction with Visual Effects has become a vital tool for teaching Visual Storytelling at Auckland University of Technology. It is part of the core papers of the Digital Design program, and is also offered as an elective for students of other programs within the Bachelor of Design degree. Decoupling the acting performance from the camera has led to a flexibility, which allows to explore different aspects of visual narratives from traditional linear approaches, to more contemporary developments with visual effects-enabled 'impossible'moves, and even unconstrained environments in form of virtual reality spaces. This has changed the possibilities to actively engage students with more exciting learning outcomes, and allows playful exploration of different visual storytelling paradigms without the necessity to set up extensive practical filming scenarios or to facilitate complicated visual effects productions.

Making Motion Capture part of our curriculum in Digital Design has also posed a range of pedagogical challenges, and requires a more careful planning of resources and timeframes in order to ensure the benefits of this approach find their way into the studiobased projects of the students.

This paper outlines a successful implementation of a Motion Capture and Visual Effects curriculum around Visual Storytelling in form of a multifaceted assignment strategy. But it also raises issues around the management of complex production scenarios in context of a tertiary teaching environment.

In future, we expect to explore additional possibilities such as virtual environment rendering using VR devices like Oculus Rift, in order to facilitate learning in the Game Design stream of the Digital Design programme at Auckland University of Technology.

\section{Contact Information}

If you have questions or suggestions regarding this document, please contact Jan Kruse at “jkruse@ aut.ac.nz”.

\section{Acknowledgements}

We wish to thank Auckland University of Technology, in particular Colab for their support.

\section{References}

Autodesk. 2009. The New Art of Virtual Moviemaking - An Autodesk Whitepaper. Autodesk.

Bordwell, D., AND ThOMPSON, K. 2008. Film art: an introduction, eighth edition ed. McGraw Hill, Boston.

Bowen, C. J. 2013. Grammar of the shot, 3rd ed ed. Focal Press, Oxford.

Corrigan, T., AND White, P. 2012. The film experience: an introduction, third edition ed. Bedford/St.Martins, Boston, Mass.

DELBRIDGE, M. 2015. Motion capture in performance: an introduction. Palgrave Pivot. Palgrave Pivot, Basingstoke, Hampshire.

HaCh, T., AND Steurer, J. 2013. A Novel RGB-Z Camera for High-quality Motion Picture Applications. In Proceedings of the 10th European Conference on Visual Media Production, ACM, New York, NY, USA, CVMP '13, 4:1-4:10.

Howland, K., Good, J., And Robertson, J. 2007. A learnercentred design approach to developing a visual language for interactive storytelling. In Proceedings of the 6th International Conference on Interaction Design and Children, ACM, New York, NY, USA, IDC '07, 45-52.

KATZ, S. D. 1991. Film Directing Shot by Shot: Visualizing from Concept to Screen, 1 edition ed. Focal Press, Studio City, CA, July.

Kolker, R. 2005. Film, Form, and Culture w/ DVD-ROM, 3 edition ed. McGraw-Hill Humanities/Social Sciences/Languages, Boston, Mass., Aug.

MCClEAN, S. T. 2007. Digital storytelling: the narrative power of visual effects in film. MIT Press, Cambridge, Mass. 
Meyers, C., And Jones, T. B. 1993. Promoting Active Learning. Strategies for the College Classroom. ERIC.

Mileski, D., Schneider, F., AND BRuegge, B. 2015. Visual storytelling. In Proceedings of the 18th European Conference on Pattern Languages of Program, ACM, New York, NY, USA, EuroPLoP'13, 12:1-12:7.

OKUn, J. A., AND ZWERMAN, S., Eds. 2010. The VES Handbook of Visual Effects: Industry Standard VFX Practices and Procedures, 1 ed. Focal Press, Burlington, MA, July.

Plemmons, D., AND Holz, D. 2014. Creating next-gen 3d interactive apps with motion control and unity3d. In ACM SIGGRAPH 2014 Studio, ACM, New York, NY, USA, SIGGRAPH '14, 24:1-24:23.

RusLi, E. M. 2015. Oculus Studio to Make Virtual-Reality Movies. Wall Street Journal,Europe (Jan.), 16.

SiJll, J. V. 2005. Cinematic Storytelling: The 100 Most Powerful Film Conventions Every Filmmaker Must Know, 7.2.2005 edition ed. Michael Wiese Productions, Studio City, CA, Aug.

Wood, A. 2007. Digital encounters. Routledge, London ; New York. 